\title{
Leveraging Optogenetic-Based Neurovascular Circuit Characterization for Repair
}

\author{
Elena Ivanova $^{1} \cdot$ Christopher W. Yee $^{1} \cdot$ Botir T. Sagdullaev $^{1}$
}

Published online: 12 January 2016

(C) The American Society for Experimental NeuroTherapeutics, Inc. 2016

\begin{abstract}
Optogenetic techniques are a powerful tool for determining the role of individual functional components within complex neural circuits. By genetically targeting specific cell types, neural mechanisms can be actively manipulated to gain a better understanding of their origin and function, both in health and disease. The potential of optogenetics is not limited to answering biological questions, as it is also a promising therapeutic approach for neurological diseases. An important prerequisite for this approach is to have an identified target with a uniquely defined role within a given neural circuit. Here, we examine the retinal neurovascular unit, a circuit that incorporates neurons and vascular cells to control blood flow in the retina. We highlight the role of a specific cell type, the cholinergic amacrine cell, in modulating vascular cells, and demonstrate how this can be targeted and controlled with optogenetics. A better understanding of these mechanisms will not only extend our understanding of neurovascular interactions in the brain, but ultimately may also provide new targets to treat vision loss in a variety of retinal diseases.
\end{abstract}

Keywords Neurovascular unit · Optogenetics · Pericyte · Amacrine cell $\cdot$ Acetylcholine

Botir T. Sagdullaev

bos2005@med.cornell.edu

1 Department of Ophthalmology, Brain and Mind Research Institute, Weill Cornell Medical College, Burke Medical Research Institute, White Plains, NY 10605, USA

\section{Introduction}

Neurological diseases possess a staggering array of etiologies, involving genetic, neuronal, and regulatory factors, to name a few. Research has largely focused on targeting specific genes or molecules for treatment, which somewhat belies the complexity of the task; there are tens of thousands of genes, which have activation patterns that differ under varying circumstances, even among cells of the same type or class. Conversely, using a cell-based approach - to repair, replace, or supplement particular classes of affected cells - has historically been limited by the difficulty of distinguishing them amongst the tremendous cell diversity present in the central nervous system (CNS) [1]. In practice, therapeutic interventions have largely eschewed both of these seemingly opposing approaches in favor of a reliance on pharmacological interventions. Unfortunately, the efficacy of the pharmacological approach can be limited by the lack of specificity of a given drug to its primary target, as well as the unintended side effects of its action on tertiary targets. In theory, an ideal approach should combine all these methods to target a specific neurological circuit, defined here as the functional unit that is necessary and sufficient to perform a specific task.

A number of groundbreaking techniques have dramatically enhanced our understanding of the circuits responsible for neurological diseases, and our ability to target them for treatment. Cre-lox technology enables the expression, deletion, or modification of specific genes, and can be targeted to specific cell types. This has been used in combination with a variety of light-activated ion channels, such as the channelrhodopsin and halorhodopsin, to genetically confer light-sensitivity to a chosen cell class. Optogenetics thus allows for the noninvasive activation or inhibition of specific cells with light, a method that can be leveraged to combat a wide range of neurological conditions. Already, the potential of optogenetic techniques 
has been demonstrated in sciatic nerve injury [2], Parkinson's disease [3], epilepsy [4], depression [5, 6], and retinal degeneration $[7,8]$. In this work, we describe a new method of using optogenetic techniques to study retinal circuit interactions between neurons and vascular cells, and show how this general approach can be potentially applied to treat neurological diseases.

\section{The Neurovascular Unit: a Fundamental Circuit}

In the classical view, the function of the CNS has been considered to be primarily driven by interactions between neurons. Consequently, many neurological diseases are viewed predominantly as neuronal impairments. Other cell classes, glial and vascular cells in particular, have been viewed as secondary components of the CNS, supporting neurons structurally and metabolically without actively influencing neural function. During the last few decades, however, it has become clear that this is not the case, and that these supporting cells, in fact, play crucial active roles [9]. Though our understanding of the individual components of the nervous system has been increasing exponentially, failing to consider fully the interactions between these diverse elements inherently limits our perspective on the physiology and pathophysiology of the $\mathrm{CNS}$, in turn limiting our ability to translate these findings to clinical applications. One of the most important outcomes of these interactions is the regulation of blood flow in the CNS.

The brain is the most metabolically demanding organ in the human body, accounting for $20 \%$ of its resting energy consumption [10]. To meet this demand, blood vessels deliver oxygen and nutrients in an activity-dependent manner, a process called functional hyperemia [11]. This hemodynamic process relies on signaling between neurons, vascular cells, and glia, operating in homeostatic balance [12]. Together, this network is known as the neurovascular unit [13, 14]. Neurological conditions that disrupt any of these neurovascular components can affect the rest of the unit, leading to a cycle of reciprocal attrition, ultimately leading to the loss of the blood-brain barrier and neuronal degeneration. Thus, a comprehensive analysis of a given neurological disease should identify the affected neurovascular unit(s) within the particular tissue, and account for the function of each neurovascular component.

Recent studies have suggested that neuronal activity can directly regulate blood flow by controlling the diameter of blood vessels [15]. The diameter of large blood vessels is controlled by smooth muscles that wrap their walls. However, the majority of neurovascular interactions take place within the extensive capillary network, which lacks both smooth muscles and central innervation. Across many parts of the brain, the diameter of these capillaries is directly regulated by specialized contractile cells, called pericytes (Fig. 1a),
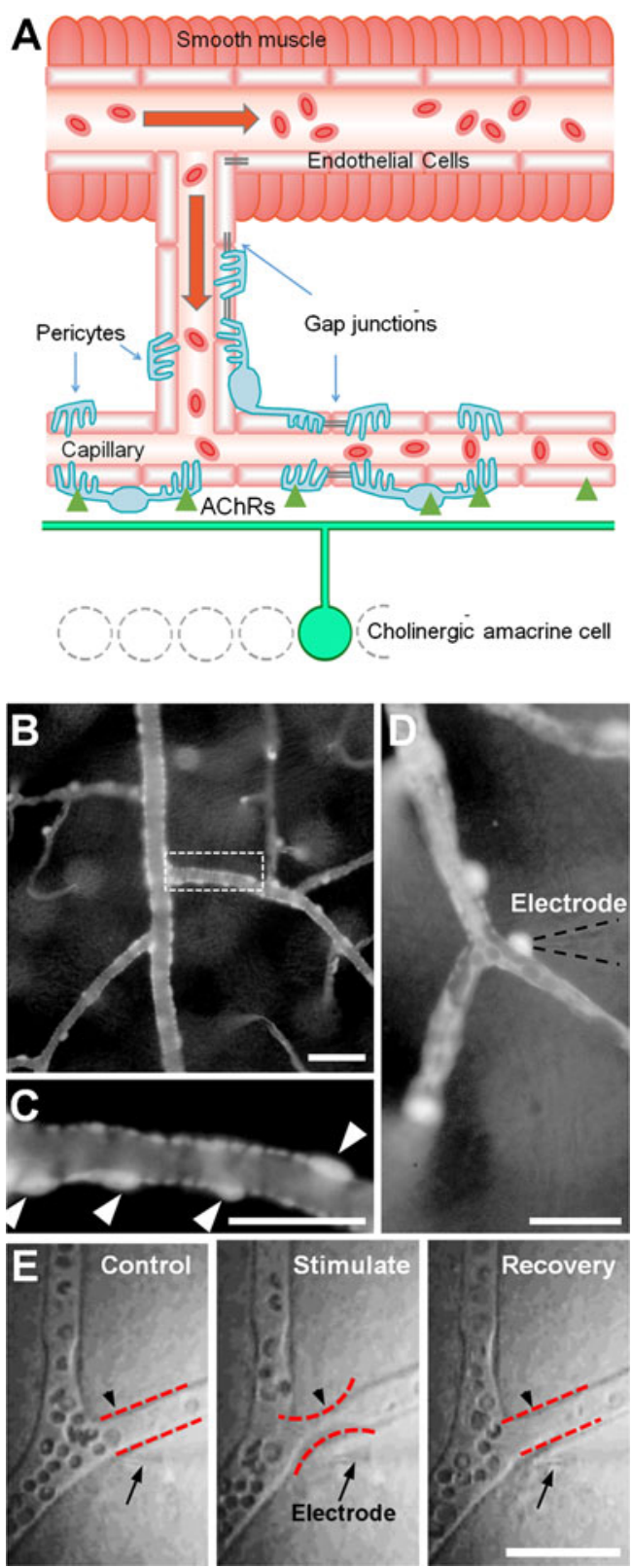

Fig. 1 Pericyte control of capillary diameter. (A) Simplified schematic of blood vessel regulation components in retina (modified from [15]). In response to light stimulation of the retina, cholinergic amacrine cells release neurotransmitter that activates acetylcholine receptors (AChRs) on pericytes and endothelial cells. Activity from an individual pericyte can spread via gap junctions across the vascular network to regulate upstream blood vessels. (B) Live fluorescent image of intact retina in NG2-DsRed mice, revealing pericyte bodies and processes. Boxed region is enlarged in $(\mathbf{C})$. White arrowheads point to individual pericyte cell bodies. (D) An example shows how a single pericyte is stimulated using an electrode (dotted lines) to assess changes in diameter of blood vessel, or its vasomotor response. (E) Focal electrical stimulation of a pericyte induces a robust and reversible vasomotor response (constriction). Scale bars $=25 \mu \mathrm{m}$

which, in turn, are controlled by local neuronal activity [15, 16]. Pericytes "tile" the surface of blood capillaries and have processes that wrap around the vessels (Fig. 1b, c). Focal 
stimulation of an individual pericyte can produce a strong vasomotor response (Fig. 1d, e). It has been shown that under ischemic conditions this response is significantly reduced, suggesting a critical role for proper neurovascular control in neurological dysfunctions [17].

Furthermore, this initially local response can spread along the neighboring pericytes to control blood flow tens of micrometers away from the site of stimulation $[15,16,18]$. This propagative nature of pericyte activity may play a critical role in both local network interactions and in signaling to regulate retinal blood flow upstream, in precapillary arterioles [17]; however, the precise mechanism remains unclear. This function would require communication not only among pericytes, but also pericytes and endothelial cells. The anatomical presence of gap junctions between pericytes, as well as endothelial cells, supports their role in intercellular interactions in the retinal blood vessels [19]. These gap junctions are comprised of connexin43-containing subunits that are shown to form connections with large conductances needed for fast signal transmission across the network of coupled cells [20-22].

\section{Neuronal Control of Blood Flow: a Role for Cholinergic Regulation}

Activity-dependent regulation of blood flow starts with neurons releasing neurotransmitters. Multiple mechanisms have been proposed for neurovascular regulation of blood vessels in healthy retina, including arachidonic acid metabolites, nitric oxide (NO), and acetylcholine (ACh) [18, 23-26]. Among numerous neurotransmitters, $\mathrm{ACh}$ is believed to play a central role. While most of the vasoactive neurotransmitters are released by variety of neurons, only ACh is selectively released by a well-characterized population of neurons - cholinergic amacrine cells $[27,28]$. The vasodilatory effect of ACh on retinal capillaries and arterioles has been well established [18, 29-31].

In the retina, $\mathrm{ACh}$ has been shown to be released by cholinergic amacrine cells in response to transients of light, both at onset and offset of stimulation [32]. The release was maximally stimulated by a flickering light [33-35], thus making $\mathrm{ACh}$ an ideal mediator of flicker-induced dilation of blood vessels (for review, see [24, 36, 37]). This unique ability to use natural stimuli to probe the function of the neural circuit makes the retina an outstanding model for studies of neurovascular function.

Retina, the most approachable part of the CNS, shares the same key elements as other parts of the CNS (Fig. 2). Beneficially, it can be readily and selectively stimulated with physiologically appropriate stimulus for assessment and, potentially, treatment. Its natural stimulant, light, could be noninvasively delivered at wide range of modalities; spectral frequencies for selective pigment stimulation, temporal frequency, and
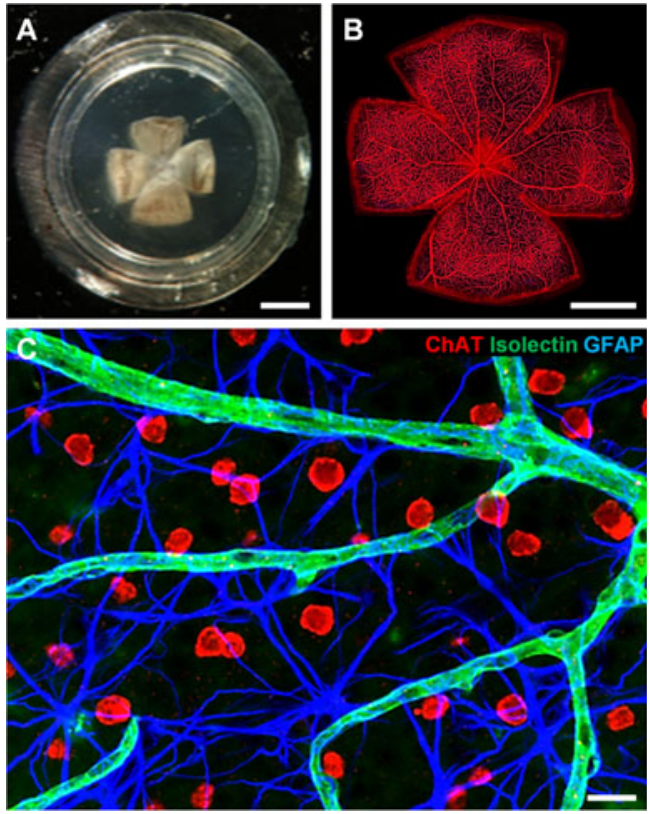

Fig. 2 Retina as a model to study function of the central nervous system in health and disease. (A) Mouse retina wholemount placed on a Millicell Biopore membrane filter. Once immersed in physiological media, filter becomes transparent. Scale bar $=2 \mathrm{~mm}$. (B) Confocal live imaging of a whole intact retina illustrating retinal vasculature stained with endothelial cell-specific marker added to perfusion media (SRH, red, modified from [39]). Scale bar $=1 \mathrm{~mm}$. (C) A magnified section of the retina illustrating individual components of the hypothetical neurovascular unit. Blood vessels (isolectin, green), neurons, cholinergic amacrine cells [choline acetyltransferase (ChAT), red], and astroglia [glial fibrillary acidic protein (GFAP), blue]. Scale bar $=25 \mu \mathrm{m}$

spatial patters combined across wide intensity ranges can all be tailored for preferential targeting of distinct visual pathways. Intact retina can be dissected without disrupting integrity of internal connections and all components of the neurovascular unit could be identified and probed [38, 39] (Fig. 2c). From a translational point of view, retina is a site of wide range of neurodegenerative, traumatic, metabolic, and vascular diseases that are naturally present in humans and could be induced with numerous treatments in animal models.

In the retina, a low tonic release of ACh was also reported [40], which may be important to maintain a tonic state of the vasculature and the basal blood flow. What is the mechanism of ACh-mediated vasodilation? The vascular endothelium of large blood vessels synthesizes the vasodilator NO during exposure to acetylcholine [41, 42]. Consistently, cholinergic vasodilation of murine retinal arterioles was also shown to be primarily mediated by $\mathrm{NO}$, since blockade of $\mathrm{NO}$ production almost completely abolished responses to ACh. When endothelial cells were damaged, there was no dilation, but vasoconstriction was present [31]. It is likely that ACh released from cholinergic cells binds to muscarinic receptors found on pericytes and endothelial cells and stimulates NO production in the endothelial cells. NO generates hyperpolarizing currents that would be expected to relax pericytes and dilate the 
capillaries [43]. Vascular diameter responses can propagate between adjacent pericytes $[15,44]$, but it is not known whether arterioles receive a signal to dilate from pericytes, or from vasoactive messengers, which reach arterioles later than they reach capillaries [17].

The retinal vasculature is composed of four distinct vascular layers (Fig. 3a). The superficial layer is located in the outer
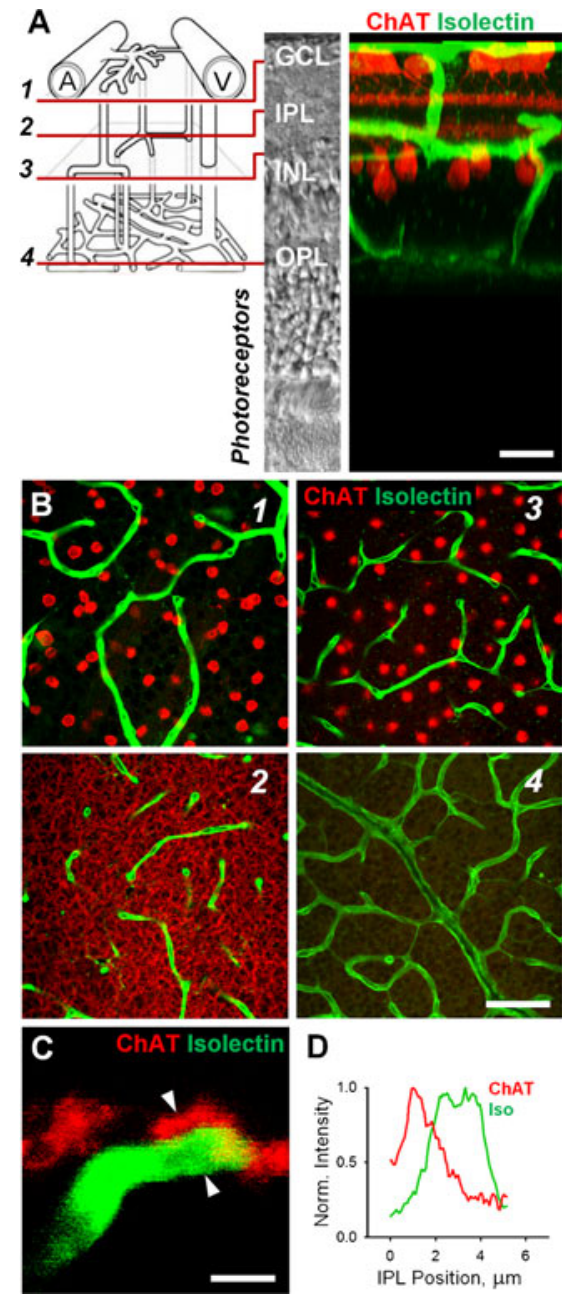

Fig. 3 Cholinergic neurovascular interaction sites in the retina. (A) Schematic of the laminar neurovascular network showing the first-order arteriole (A) and venule (V) and the connectivity of the (1) superficial, (2) intersublaminar, (3) intermediate, and (4) deep vascular layers (left) and their locations within the retina (middle). Blood vessels are labeled with isolectin (right, green). In red are cell bodies and the processes of cholinergic amacrine cells, a source of neuro- and vasomodulator, acetylcholine. $\mathrm{GCL}=$ ganglion cell layer; IPL $=$ inner plexiform layer; $\mathrm{INL}=$ inner nuclear layer; OPL = outer plexiform layer. Drawing is modified from [37]. (B) Confocal images of the (1) superficial, (2) intersublaminar, (3) intermediate, and (4) deep vascular layers, labeled with isolectin (green) and choline acetyltransferase (ChAT; red). Numbers are vascular layers as in (a). Panel (B2) illustrates potential sites of neurovascular interactions between cholinergic cells blood vessels. Scale bar $=50 \mu \mathrm{m}$. (c) Z-stack reconstruction from a magnified area in panel (B2). (D) Intensity profiles across an imaginary line between arrowheads in (c). Scale bar $=50 \mu \mathrm{m}$ in (A) and (B). Scale bar $=5 \mu \mathrm{m}$ in (C). (Modified from [46]) boundary of the ganglion cell layer and includes venules and arterioles. Three deeper vascular layers (deep, intermediate, and intersublaminar) are intercalated between neuronal synapses in the outer and inner plexiform layers and are comprised of capillaries (Fig. 3a-d). Because the synaptic release of ACh was found in the inner plexiform layer at both the cholinergic bands we hypothesized that the intersublaminar blood vessel layer recently characterized by our group could be the site of neurovascular interactions (Fig. 3) [32, 45, 46]. This additional layer of blood vessels was found to coincide with the OFFChAT band. It was equally prominent at all retinal poles and eccentricities, comprising $\sim 7-8 \%$ of the total length of horizontally running blood vessels. As retinal vasculature lacks autonomic control, and shows an efficient local regulation $[47,48]$, the function of vascular cells may be modulated by neurons in this region. Pericytes are the contractile cells of the vasculature [15], and are more dense in retinal blood vessels than anywhere in the brain [49]. They express functional receptors for a variety of synaptic transmitters, which can cause contraction or relaxation $[15,18,50]$. For example, application of ACh alters the membrane potential of pericytes, leading to cell contraction and constriction of the blood vessel [18, 31]. Thus, it is possible that the cholinergic cells may modulate blood vessel diameter via pericytes to modulate blood flow, though further investigation is needed to confirm this. Several recent studies also indicate that glial cells can act as intermediaries in signaling from neurons to blood vessels $[25$, 51]. These interactions can be disrupted during retinal disease, which provides a strong impetus for future studies to characterize the retinal neurovascular unit.

\section{Optogenetic Control of a Defined Neural Circuit: a Strategy for Functional Repair}

$\mathrm{ACh}$ is a potent vasodilator. Muscarinic ACh receptors (type M3) are present in retinal vasculature and are essential for vasodilation [29, 31, 52]. Indeed, application of $100 \mu \mathrm{M}$ carbachol, a nondegradable analogue of $\mathrm{ACh}$, resulted in significant dilation in both capillaries and precapillary arterioles (see Fig. 5a, b). However, global application of receptor agonist is a valuable tool but may differ from synaptic release of neurotransmitter. This is, in part, due to lack of spatial and temporal precision. To determine the precise role of cholinergic amacrine cells in vasomotor control, we have designed a method to selectively activate neurotransmitter release from the cholinergic neurons in the retina (Fig. 4). In our approach, we chose to target cholinergic amacrine cells, which represent neuronal cell types whose activity can directly affect the vasomotor response. Importantly, cholinergic amacrine cells are known to be compromised at numerous retinal diseases and represent a valuable model in which to study the pathological events, as well as serve a potential target for treatment $[53$, 
A
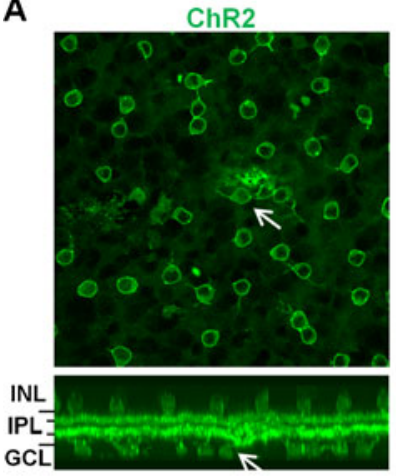

B

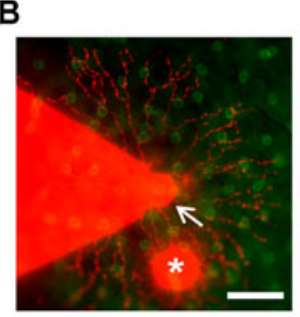

C
ChR2 ChAT Alexa
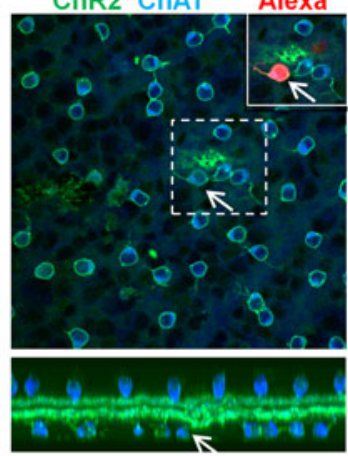

ChR2-Evoked Potentials

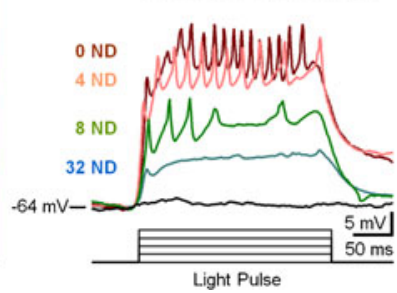

ChR2 ChAT Alexa

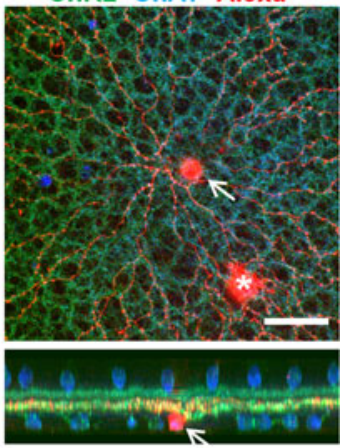

ChR2-Evoked Currents

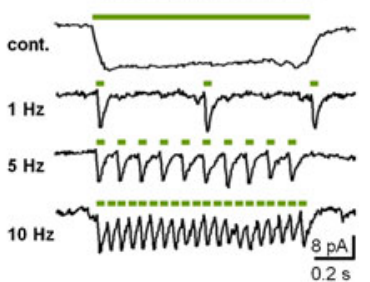

Fig. 4 Channelrhodopsin-2 (ChR2)-assisted probing of the cholinergic pathways in living mammalian retina. (A) Confocal images in choline acetyltransferase (ChAT)-channelrhodopsin-2 (ChR2)-YFP mouse retina showing selective and robust expression of $\mathrm{ChR} 2$ (green, left) in all ChAT-positive starburst amacrine cells in ganglion cell layer (blue, middle) and their processes in the inner plexiform layer (IPL, right). As shown in Z-stack reconstructions of the IPL, both ON and OFF ChATpositive cells contain ChR2. Arrows identify the cell targeted for recording. (B) In living retinal wholemount, the green fluorescent proteinpositive ChR2-expressing cells were targeted for electrophysiological recordings and backfilled with Alexa 568 included in recording pipette (arrow, red). Note a characteristic starburst appearance of ChAT-positive amacrine cell [see also (A), right]. An asterisk indicates a Muller cell damaged during the recording procedure. (C) Spiking activity (left) and excitatory currents (right) from cell in (B) in presence of synaptic blockers (LAP4, NBQX). Flashing spots of light of varying intensity and frequency produce characteristic sustained and nondesensitizing ChR2-driven activity. Neutral density (ND) values represent the ND filter attenuation of light from the light source. In right panel, the number represents the frequency in $\mathrm{Hz}$ of a flashing 50-ms-long spot of light. Scale bars $=40 \mu \mathrm{m}$
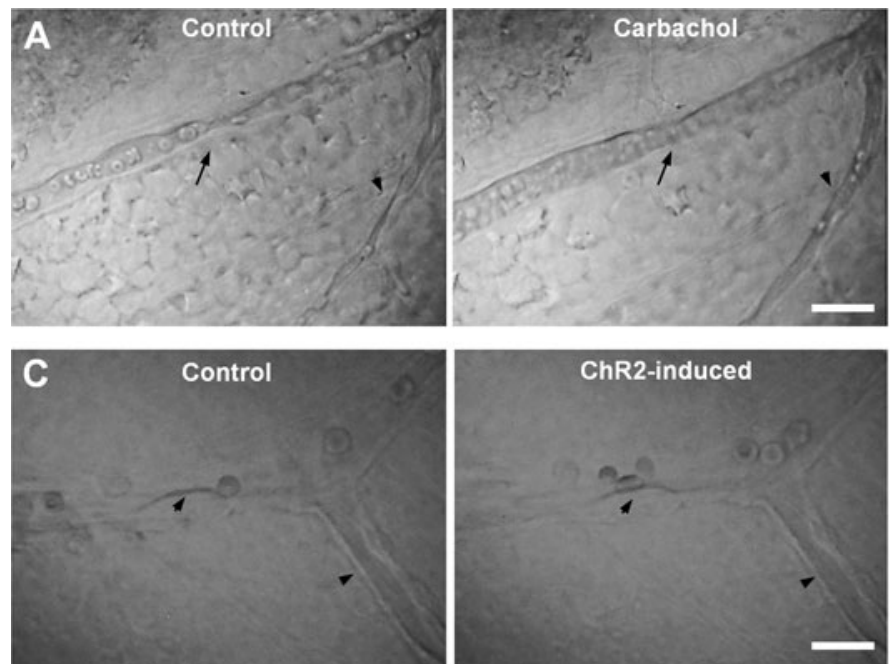

B

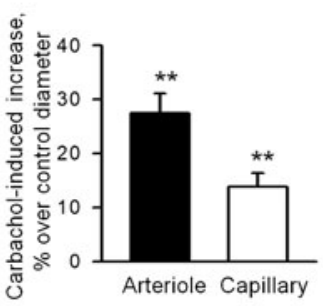

D

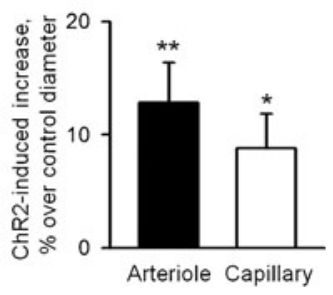

Fig. 5 Channelrhodopsin-2 (ChR2)-assisted light activation of cholinergic amacrine cells produces dilation of capillaries and precapillary arterioles. (A) Cholinergic receptor activation leads to dilation of capillaries and arterioles. Bath application of with acetylcholine analog carbachol $(100 \mu \mathrm{M})$ leads to dilation of both capillaries (arrowhead) and precapillary arterioles (arrow). (B) Quantification of vasomotor response across 40 samples showing consistent and significant changes in diameter following activation of acetylcholine receptors in intact wholemount retina. Error bars $\pm \mathrm{SEM} ;{ }^{*} p<0.001$, paired $t$ test. (C) Vasomotor response was measured when photoreceptor inputs were blocked by a mix of NBQX and L-AP4. Arrowhead points to region of dilation, allowing passage of a blood cell. (D) Quantification of vasomotor response across 15 samples each. Paired $t$ test $p=0.01$ and $p=0.03$ for arteriole and capillary, respectively. Error bars \pm SEM. Scale bars $=30 \mu \mathrm{m}$ 
54]. We have generated a mouse line in which a light-sensitive ion channel, channelrhodopsin-2 (ChR2), is genetically expressed in all cholinergic amacrine cells, under choline acetyltransferase promoter (ChAT; Fig. 4). Utilizing the Cre/lox system, we crossed homozygous ChAT-Cre mice [B6;129S6Chattm2(cre)Lowl/J, stock \#006410; The Jackson Laboratory, Bar Harbor, ME, USA] with loxP-flanked ChR2-YFP homozygous reporter mice [B6;129S-Gt(ROSA)26Sortm32(CAGCOP4*H134R/EYFP)Hze/J, stock \#012569; The Jackson Laboratory]. In our experiments, we used heterozygous ChAT-ChR2-YFP animals. In these mice, ChR2-YFP expression overlapped $100 \%$ with ChAT immunoreactivity, suggesting that all cholinergic cells, and only cholinergic cells, express the construct (Fig. 4a). ChR2 is a transmembrane ion channel, and, indeed, its expression was localized to the membrane of the cholinergic cells, unlike ChAT, which is evenly distributed in the cytoplasm of cholinergic amacrine cells (Fig. 4a). Next, we confirmed the function of ChR2 in these cells, by stimulating cholinergic amacrine cells with light, in the presence of synaptic blockers to isolate ChR2-induced activity from photoreceptor activity. Patch-clamp electrophysiological recordings were performed in identified cholinergic amacrine cells (Fig. 4b, c). Both membrane potential and current recordings from cholinergic amacrine cells in ChATChR2-YFP mice show activation threshold and temporal activity profiles consistent with a ChR2-driven response [55]. Next, we tested if selective stimulation of cholinergic cells with ChR2-induced depolarization in the absence of synaptic inputs would produce vasomotor response routinely seen during agonist-driven cholinergic activation. Consistent with this, ChR2-assisted light activation of cholinergic amacrine cells produced dilation of both capillaries and precapillary arterioles (Fig. 5). To our knowledge, this is one of the first approaches of the use of optogenetics to dissect neurovascular interactions in the CNS.

\section{Summary}

Optogenetic techniques are a valuable set of tools for studying mechanisms of the CNS. As we have shown here, optogenetics can be used to activate a specific component of a given neural circuit, in order to determine its particular function. The retina is a valuable model for this purpose, owing to its well-characterized structure and in vivo accessibility (i.e., it can be stimulated noninvasively with light through the pupil of the eye). Naturally, these characteristics have also made the retina the quintessential model for the therapeutic potential of optogenetics. Several groups have shown that, following the loss of photoreceptors, light-sensitivity can be reintroduced into remaining retinal neurons using optogenetic techniques $[7,8]$. A similar approach could be applied to other retinal diseases, particularly those with known cell-based dysfunctions. There is accumulating evidence that diabetic retinopathy compromises distinct components of the neurovascular unit, such as astrocytes, pericytes, and cholinergic amacrine cells $[54,56]$; optogenetics could target these cells to supplement their health and function.

Acknowledgments This work was supported by the Juvenile Diabetes Research Foundation (B.T.S.) and the Goldsmith Foundation (E.I.)

Required Author Forms Disclosure forms provided by the authors are available with the online version of this article.

\section{References}

1. Stevens CF. Neuronal diversity: too many cell types for comfort? Curr Biol 1998;8:R708-R710.

2. Bryson JB, Machado CB, Crossley M, et al. Optical control of muscle function by transplantation of stem cell-derived motor neurons in mice. Science 2014;344:94-97.

3. Steinbeck JA, Choi SJ, Mrejeru A, et al. Optogenetics enables functional analysis of human embryonic stem cell-derived grafts in a Parkinson's disease model. Nat Biotechnol 2015;33:204-209.

4. Zhao M, Alleva R, Ma H, Daniel AG, Schwartz TH. Optogenetic tools for modulating and probing the epileptic network. Epilepsy Res 2015;116:15-26.

5. Ramirez S, Liu X, MacDonald CJ, et al. Activating positive memory engrams suppresses depression-like behaviour. Nature 2015;522:335-339.

6. Friedman AK, Walsh JJ, Juarez B, et al. Enhancing depression mechanisms in midbrain dopamine neurons achieves homeostatic resilience. Science 2014;344:313-319.

7. Bi A, Cui J, Ma YP, et al. Ectopic expression of a microbial-type rhodopsin restores visual responses in mice with photoreceptor degeneration. Neuron 2006;50:23-33.

8. Lagali PS, Balya D, Awatramani GB, et al. Light-activated channels targeted to ON bipolar cells restore visual function in retinal degeneration. Nat Neurosci 2008;11:667-675.

9. Newman EA. Glial cell regulation of neuronal activity and blood flow in the retina by release of gliotransmitters. Philos Trans R Soc Lond B Biol Sci 2015;370.

10. Attwell D, Laughlin SB. An energy budget for signaling in the grey matter of the brain. J Cereb Blood Flow Metab 2001;21:1133-1145.

11. Roy CS, Sherrington CS. On the regulation of the blood-supply of the brain. J Physiol 1890;11:85-158.

12. Stanimirovic DB, Friedman A. Pathophysiology of the neurovascular unit: disease cause or consequence? J Cereb Blood Flow Metab 2012;32:1207-1221.

13. Abbott NJ, Ronnback L, Hansson E. Astrocyte-endothelial interactions at the blood-brain barrier. Nat Rev Neurosci 2006;7:41-53.

14. Neuwelt EA, Bauer B, Fahlke C, et al. Engaging neuroscience to advance translational research in brain barrier biology. Nat Rev Neurosci 2011;12:169-182.

15. Peppiatt CM, Howarth C, Mobbs P, Attwell D. Bidirectional control of CNS capillary diameter by pericytes. Nature 2006;443:700-704.

16. Puro DG. Physiology and pathobiology of the pericyte-containing retinal microvasculature: new developments. Microcirculation 2007;14:1-10.

17. Hall CN, Reynell C, Gesslein B, et al. Capillary pericytes regulate cerebral blood flow in health and disease. Nature 2014;508:55-60.

18. Wu DM, Kawamura H, Sakagami K, Kobayashi M, Puro DG. Cholinergic regulation of pericyte-containing retinal microvessels. Am J Physiol Heart Circ Physiol 2003;284:H2083-H2090. 
19. Sagaties MJ, Raviola G, Schaeffer S, Miller C. The structural basis of the inner blood-retina barrier in the eye of Macaca mulatta. Invest Ophthalmol Vis Sci 1987;28:2000-2014.

20. Janssen-Bienhold U, Dermietzel R, Weiler R. Distribution of connexin 43 immunoreactivity in the retinas of different vertebrates. J Comp Neurol 1998;396:310-321.

21. Bobbie MW, Roy S, Trudeau K, Munger SJ, Simon AM. Reduced connexin 43 expression and its effect on the development of vascular lesions in retinas of diabetic mice. Invest Ophthalmol Vis Sci 2010;51:3758-3763.

22. Oku H, Kodama T, Sakagami K, Puro DG. Diabetes-induced disruption of gap junction pathways within the retinal microvasculature. Invest Ophthalmol Vis Sci 2001;42:1915-1920.

23. Kur J, Newman EA, Chan-Ling T. Cellular and physiological mechanisms underlying blood flow regulation in the retina and choroid in health and disease. Prog Retin Eye Res 2012;31:377406.

24. Newman EA. Functional hyperemia and mechanisms of neurovascular coupling in the retinal vasculature. J Cereb Blood Flow Metab 2013;33:1685-1695.

25. Metea MR, Newman EA. Glial cells dilate and constrict blood vessels: a mechanism of neurovascular coupling. J Neurosci 2006;26:2862-2870.

26. Allt G, Lawrenson JG. Pericytes: cell biology and pathology. Cells Tissues Organs 2001;169:1-11.

27. Hayden SA, Mills JW, Masland RM. Acetylcholine synthesis by displaced amacrine cells. Science 1980;210:435-437.

28. Famiglietti EV, Jr. 'Starburst' amacrine cells and cholinergic neurons: mirror-symmetric on and off amacrine cells of rabbit retina. Brain Res 1983;26:138-144.

29. Benedito S, Prieto D, Nielsen PJ, Nyborg NC. Role of the endothelium in acetylcholine-induced relaxation and spontaneous tone of bovine isolated retinal small arteries. Exp Eye Res 1991;52:575579.

30. Schonfelder U, Hofer A, Paul M, Funk RH. In situ observation of living pericytes in rat retinal capillaries. Microvasc Res 1998;56:2229.

31. Gericke A, Sniatecki JJ, Goloborodko E, et al. Identification of the muscarinic acetylcholine receptor subtype mediating cholinergic vasodilation in murine retinal arterioles. Invest Ophthalmol Vis Sci 2011;52:7479-7484

32. Masland RH, Livingstone CJ. Effect of stimulation with light on synthesis and release of acetylcholine by an isolated mammalian retina. J Neurophysiol 1976;39:1210-1219.

33. Massey SC, Neal MJ. The light evoked release of acetylcholine from the rabbit retina iN vivo and its inhibition by gammaaminobutyric acid. J Neurochem 1979;32:1327-1329.

34. Massey SC, Redburn DA. Light evoked release of acetylcholine in response to a single flash: cholinergic amacrine cells receive ON and OFF input. Brain Res 1985;328:374-377.

35. O'Malley DM, Masland RH. Responses of the starburst amacrine cells to moving stimuli. J Neurophysiol 1993;69:730-738.

36. Garhofer G, Zawinka C, Resch H, Kothy P, Schmetterer L, Dorner GT. Reduced response of retinal vessel diameters to flicker stimulation in patients with diabetes. Br J Ophthalmol 2004;88:887-891.

37. Kornfield TE, Newman EA. Regulation of blood flow in the retinal trilaminar vascular network. J Neurosci 2014;34:11504-11513.
38. Ivanova E, Toychiev AH, Yee CW, Sagdullaev BT. Optimized protocol for retinal wholemount preparation for imaging and immunohistochemistry. J Vis Exp 2013; e51018.

39. Toychiev AH, Sagdullaev B, Yee CW, Ivanova E, Sagdullaev BT. A time and cost efficient approach to functional and structural assessment of living neuronal tissue. J Neurosci Methods 2013;214: 105-112.

40. Lipton SA. Spontaneous release of acetylcholine affects the physiological nicotinic responses of rat retinal ganglion cells in culture. J Neurosci 1988;8:3857-3868.

41. Furchgott RF, Zawadzki JV. The obligatory role of endothelial cells in the relaxation of arterial smooth muscle by acetylcholine. Nature 1980;288:373-376.

42. Palmer RM, Ferrige AG, Moncada S. Nitric oxide release accounts for the biological activity of endothelium-derived relaxing factor. Nature 1987;327:524-526.

43. Sakagami K, Kawamura H, Wu DM, Puro DG. Nitric oxide/cGMPinduced inhibition of calcium and chloride currents in retinal pericytes. Microvasc Res 2001;62:196-203.

44. Puro DG. Retinovascular physiology and pathophysiology: new experimental approach/new insights. Prog Retin Eye Res 2012;31:258-270.

45. Koulen P. Vesicular acetylcholine transporter (VAChT): a cellular marker in rat retinal development. Neuroreport 1997;8:2845-2848.

46. Ivanova E, Toychiev AH, Yee CW, Sagdullaev BT. Intersublaminar vascular plexus: the correlation of retinal blood vessels with functional sublaminae of the inner plexiform layer. Invest Ophthalmol Vis Sci 2014;55:78-86

47. Ye XD, Laties AM, Stone RA. Peptidergic innervation of the retinal vasculature and optic nerve head. Invest Ophthalmol Vis Sci 1990;31:1731-1737.

48. Delaey C, Van De Voorde J. Regulatory mechanisms in the retinal and choroidal circulation. Ophthalmic Res 2000;32:249-256.

49. Sims DE. Diversity within pericytes. Clin Exp Pharmacol Physiol 2000;27:842-846.

50. Wu DM, Kawamura H, Li Q, Puro DG. Dopamine activates ATPsensitive $\mathrm{K}+$ currents in rat retinal pericytes. Vis Neurosci 2001;18: 935-940.

51. Koehler RC, Gebremedhin D, Harder DR. Role of astrocytes in cerebrovascular regulation. J Appl Physiol (1985) 2006;100:307317.

52. Hoste AM, Andries LJ. Contractile responses of isolated bovine retinal microarteries to acetylcholine. Invest Ophthalmol Vis Sci 1991;32:1996-2005.

53. Engerman RL, Kern TS. Retinopathy in animal models of diabetes. Diabetes Metab Rev 1995;11:109-120.

54. Gastinger MJ, Singh RS, Barber AJ. Loss of cholinergic and dopaminergic amacrine cells in streptozotocin-diabetic rat and Ins2Akita-diabetic mouse retinas. Invest Ophthalmol Vis Sci 2006;47:3143-3150.

55. Zhao S, Ting JT, Atallah HE, et al. Cell type-specific channelrhodopsin-2 transgenic mice for optogenetic dissection of neural circuitry function. Nat Methods 2011;8:745-752.

56. Antonetti DA, Klein R, Gardner TW. Diabetic retinopathy. N Engl J Med 2012;366:1227-1239. 